\title{
The Effect of Designed Educational Program on Midwives Knowledge and Practice Regarding Post Natal Sepsis Management in Governmental Hospitals (Jan 2020), River Nile State, Sudan
}

Manahil Omer Mohemmed ${ }^{1 *}$, Widad Ibrahim², A. Gadir ${ }^{3}$, A. Moula ${ }^{3}$

${ }^{1}$ College of Applied Medical Sciences, University of Bisha, 255, Bisha 67714, Saudi Arabia

${ }^{2}$ College of Applied Medical Sciences, Taif University, Al Huwaya, Taif 26571, Saudi Arabia

${ }^{3}$ Rented from University of Bahri, Sudan

DOI: $10.36348 / \mathrm{sjnhc} .2020 . \mathrm{v} 03 \mathrm{i} 01.004$
*Corresponding author: Manahil Omer Mohemmed

Abstract

Background: Postnatal sepsis is one of the problems that threaten the lives of mothers, although it can be avoided by taking the necessary precautions. The nurse and midwife represent the cornerstone in preventing and treating infections during the postpartum period. Study Design: This semi-experimental study Sudan, River Nile state to evaluate the effectiveness of designed guidelines for midwives regarding postnatal sepsis management, during the period March 2017 to January 2020. Sample Size: The study included 73 midwives. Data Collection Tools: Data were collected in two stages using a standard questionnaire and a list of observations before and after the training program. Data Analysis: The data were analyzed by a computer software program (SPPS) version 23. The result showed that the level of knowledge is improved significantly after guidelines interventions, where Nursing assessment and management 20.8\% had bad practice while this is improved $82.6 \%$ very good practice. only $12.5 \%$ of respondents scored very good Skills full of maintaining vulval hygiene and Maternal hygiene, which change to $100 \%$ very good score after interventions. concerning counseling regarding prevention and management $29.2 \%$ considered as bad practice changed to $91.3 \%$ Very Good Practice after interventions of guidelines. Conclusion: The study concluded that the majority of nurses midwives had high knowledge score in the post-test compared with pre-test and they demonstrated good practice which indicated that the postpartum infection management training guidelines program was effective.

Keywords: Postnatal sepsis, (SPPS), midwives.

Copyright @ 2020: This is an open-access article distributed under the terms of the Creative Commons Attribution license which permits unrestricted use, distribution, and reproduction in any medium for non-commercial use (NonCommercial, or CC-BY-NC) provided the original author and source are credited.

\section{INTRODUCTION}

Eeach year more than 500,000 women between the ages of 15 and 49 die of causes related to pregnancy and childbirth, and more than half occur in Africa according to a recent WHO estimation [1]. The vast majority of these deaths are preventable. Researchers also estimate that more than 40 percent of pregnant women experience obstetric disorders that are not immediately fatal [2]. Approximately 15 percent of all births are complicated by a potentially fatal condition that requires emergency care [3].

The five most important direct causes of maternal mortality in developing countries are hemorrhage, sepsis, unsafe abortion, eclampsia, and obstructed labor. Together these causes account for more than two-thirds of maternal mortality in the world
The second leading cause of maternal mortality, sepsis, is estimated to cause 15 percent of all maternal deaths worldwide [3]. Puerperal infections are caused by the transfer of an infectious agent from the cervix or vagina to the uterus during labor or pelvic examination or by transfer of bacteria from skin, nostrils, and perineum by contaminated fingers or instruments. The risk of puerperal sepsis is higher for women with sexually transmitted and other infections, premature rupture of membranes, retained products of conception, diabetes, cesarean or other operation, postpartum hemorrhage, anemia, poor nutritional status, history of previous complications of labor, and poor infection control [4].

A study conducted about Maternal Mortality in Ribat University Hospital, Khartoum, Sudan: Seven years of experience concluded that: Maternal mortality rate was 51:100000 live births. Pregnancy-induced hypertension constituted one-half of the causes of 
maternal deaths, followed by haemorrhage and septicaemia [5].

A cross-sectional community-based study carried out in Kassala, eastern Sudan during February through March 2012 to describe the postpartum activities (breastfeeding, perineal hygiene, use and time of family planning, how they aid locia and induce uterine involution), postpartum care (postnatal check-up by health provider) and the association of postpartum care with socio-demographic characteristics (age, education... etc.), parity and antenatal care. Results out of 300 interviewed women $55.7 \%$ (167\300) didn't receive any postnatal check-up till they completed 6 weeks from their last delivery. 63\% (189\300) applied herbal material on the episiotomy wounds and $92 \%$ (2761300) induce drainage of lochia and uterine involution by drinking various materials, while $8 \%$ (24\300) applied hot compresses andlor sat on the hot water and salt solution. The age didn't significantly influence postnatal care (P-value 0.1); however, parity, education, and antenatal care coverage were statistically varied with postnatal care usage $(\mathrm{P}$ value $=0.001,0.00$ and 0.001 respectively). Conclusion, In Summary, of this paper calls for urgent medical attention in the postpartum period. Thus the current study aimed to describe postnatal care and activities among women and expected to provide the key persons and program managers with fundamental data that might improve the quality of maternity care.

\section{JUSTIFICATION}

Sudan was one of eleven countries that are responsible for $65 \%$ of global maternal deaths according to a recent World Health Organization (WHO) estimate. To reduce this high maternal mortality rate improving the provision of emergency obstetric care (Emoc) in all health facilities, expanding midwifery training and coverage especially in rural areas is recommend [3].

To support the upgrading of midwifery skills by strengthening maternal and newborn health services, a set of midwifery training and developing modules. The need of this modules should be identified by the midwives and nurses who attended the program

This study had been chosen to help in reducing the incidence of puerperal sepsis by teaching nurses and midwives who are responsible for giving care and educate the women within postnatal period.

\section{OBJECTIVES}

\section{General Objective}

To study The Effectiveness of Designed Guidelines for nurses Regarding postnatal sepsis Management in Governmental Hospitals, at River Nile State, Sudan in 2019.

\section{SPECIFIC OBJECTIVES}

1. To determine the level of knowledge of nurses Regarding postnatal sepsis Management.

2. To assess the various activities, which are done by nurses Regarding postnatal sepsis Management.

3. To determine the extent of change for knowledge and practice of nurses after the implementation of the Designed Guidelines training program.

\section{METHODOLOGY STUDY DESIGN}

A semi-experimental pre-posttest was adopted to study the effect of Designed Guidelines Regarding postnatal sepsis Management in Governmental Hospitals on knowledge and practice for nurses and midwives. The study sought to assess the exact knowledge and information midwives have regarding postpartum postnatal sepsis before the intervention and to assess knowledge and evaluate their skills after the implementation of the program.

\section{STUDY AREA}

The study was conducted at River Nile State. Which is situated north of Khartoum state. The area of the locality is about 122.123 kilometer2, located between 16- 22 north longitudes and 30- 32 east latitude. The population was about 1,511,442.

It consists of 7 localities:

- Damir locality (area 32 thousand square kilometers, population 284 thousand people)

- Atbara locality (3.8 thousand square kilometers, 131 thousand people)

- Shendi locality (268 thousand people)

- Berber locality (160 thousand people)

- Al-Mutama locality (12 thousand square kilometers, 151 thousand inhabitants)

- Abu Hamad locality (38 thousand square kilometers, 80 thousand people)

- The locality of the lake

The total numbers of hospitals at River Nile State are 38with 2305 beds only 438 of it for obstetric and gynecological cases [6].

\section{Study Setting}

The study was conducted at Al Damir hospital it's capacity 126 beds, Atbara Teaching hospital it's capacity 362 beds, Alzeidab Rural hospital it's capacity 75 beds, AL Salam, it's capacity 70 beds University hospital and Atbara midwifery school center [6].

\section{STUDY POPULATION}

The study population consists of nurses working at hospitals in midwifery sections, Nurse midwives and midwives who were subjects that possess some of the common characteristics and directly 
involved in the provision of maternal services and postnatal care and home visitors in urban and rural, areas in River Nile state, Sudan.

Total numbers of nurse-midwives 73 .

SAMPLE SIZE: Was the total coverage.

\section{RESULT}

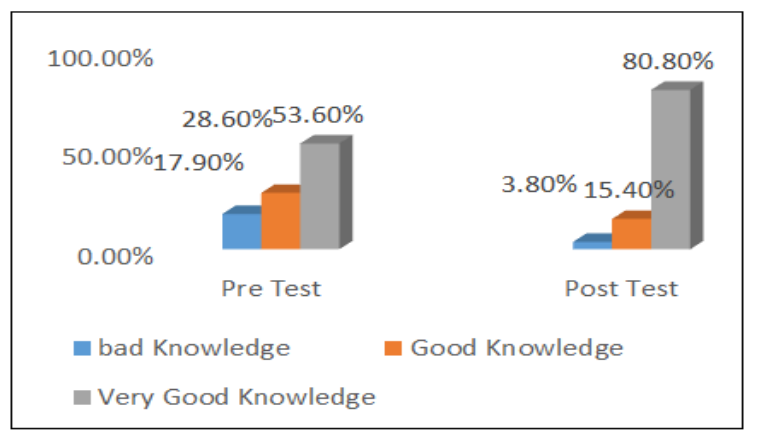

Fig-1: Distribution of the nurses midwives, knowledge regarding advice should be given to the mother generally

Nurses midwives should be knowledgeable about health problems experienced by the mother during postpartum to take suitable management early as possible, Concerning knowledge regarding health problems experienced by the mother during postpartum period, the current study illustrated that $19.2 \%$ of nurses midwives had poor score of knowledge in the pretest, while most of them had very good score of knowledge in posttest. This finding is in the agreement with $\mathrm{Li}$ XF, Fortney JA, kotelchuck M, Glover LH [7] they mentioned that the first $24 \mathrm{~h}$ postpartum and the first postpartum week is the high risk of postpartum deaths, and the risk remains significant until the second week after delivery. In developing countries, hemorrhage, pregnancy-induced hypertension complications, and obstetric infection are commonest causes of postpartum deaths.

Comparison of effects of the interventional program on nurses' knowledge regarding definitions of puerperal sepsis they assigned that more than sixty 60 $\%$ of nurses midwives and $100 \%$ of TBAs in pretest were having poor knowledge score wherein posttest great majority $72 \%$ of the nurses' midwives and $77 \%$ TBAs improved their knowledge score. The importance of this point is mentioned in a study done by the Medical Intensive Care Unit, Hamad General Hospital, Hamad Medical Corporation, Doha, Qatar [8]. Which stated that a major limitation to effective sepsis management is inadequate medical staffing and poor knowledge and awareness of sepsis which may not manifest clear cut signs and expertise to identify it is required of course, knowledge regarding Clinical signs of puerperal sepsis is so important if the caregiver not detect its good case will be hidden and not get suitable management so increase maternal morbidity and mortality.
The present study stated that the majority of the study sample had a poor score of knowledge about common Clinical signs of puerperal sepsis during the postpartum period. However, most of the participants had a very good score of knowledge in the post-test after receiving the program. This outcome is the same line with the study of Labib, Ahmed [8], they reported that Septic patients will present differently with some having subtler signs and symptoms. Moreover, Cunha, Marcia Reign [9] studied 89 medical records after analyzed, 62 of them with incomplete information. In 11 , there was at least one of the signs and symptoms suggestive of infection. A study was done by Burlinson, C. E. G et al., [10] stated that the Recognition and treatment of maternal sepsis are often delayed due to the physiological adaptations of pregnancy and vague or absent signs and symptoms during its initial presentation.

Nurses should play an important role in avoiding risk factors that may lead to postnatal sepsis. The present study revealed that $37.5 \%$ of studied participants in the pre-test were having poor knowledge regarding risk factors which could be identified in history taking, wherein the post-test majority of the participants had a high score of knowledge. In partial agreement with. LABIB, Ahmed [8] study suggested over 19 million cases and 5 million sepsis-related deaths annually. Addressing the challenge, the World Health Assembly of the World Health Organization (WHO) passed a resolution on better prevention, diagnosis, and management of sepsis.

Regarding risk factors for puerperal sepsis which could be identified by physical examination, the present study documented that, there is a significant improvement of the participants' knowledge after the implementation of the interventions compared with before intervention. These findings contradicted with Labib, Ahmed [8] they revealed that. The cornerstones of sepsis care remain early recognition, adoption of a systematic evidence-based bundle of care, and timely escalation to a higher level of care. The sepsis needs to restore physiological variables within one hour of recognition this could only be achieved if health workers have good knowledge and skills, and technical competence to provide such prevention and management.

WHO consolidated guideline on essential intrapartum care stated that problems related to infection control in developing countries include bad antibiotics prescribing practices, so about advice provided to the mother regarding using antibiotics prescribed by doctor, the present study showed more than $46.2 \%$ of the studied sample had poor knowledge before intervention, while nearly $66.7 \%$ very good score of knowledge was reported after intervention. Only $(9.1 \%)$ of traditional midwives scored very good knowledge in the post-test after receiving the training program. 
This comes in the same line with Simon, E. G., Laffon, M [11] they stated that Infection of perineal scar justifies an oral broad-spectrum antibiotics, in addition to local nursing (professional consensus).also a study by Maclean, Gaynor D [12] reported the early detection of infection and the timely use of antibiotics also reduce maternal morbidity and mortality.

The postnatal sepsis can be prevented, so skilled midwifery care that is based on the prevention of infection and complications is essential. The current study revealed that there was a significant improvement regarding knowledge of nurses about advice provides to the mother generally to prevent puerperal sepsis after receiving the training program. This finding is consistent with Seyedeh et al., [13] they reported that there are no statistical differences between pre and posttest.

Care during the postpartum period provides opportunities to health workers to detect life-threatening conditions which may result from puerperal sepsis as early as possible Concerning knowledge regarding dangerous signs life-threatening conditions which may result from puerperal sepsis during postpartum period, the present study stated that highly significant score of knowledge was recorded after intervention compared with before intervention, In partial agreement with this findings Labib, Ahmed [8] they revealed that Pregnancy and childbirth are risk factors for sepsis. Multi-organ failure and death can result from puerperal sepsis. Sepsis is the direct and leading cause of maternal mortality.

This study identified that more than half of the studied nurses had a good score of knowledge regarding advice provided to the mother concerning $\mathrm{C} / \mathrm{S}$ wound in the pretest, while these scores changed to an adequate level of knowledge in the posttest after receiving the training program with highly statistically significant differences. Robert P. Harris demonstrated convincingly that death from infection after cesarean section could be reduced significantly by operating early, rather than after several days of labor, by using an aseptic surgical technique, and by closing the uterine incision [14].

The present study Concerning knowledge regarding advice should be provided to the mother about infection control measurements during postpartum period $(26.1 \%)$ of nurses midwives and $(40.0 \%)$ of TBAs had poor score of knowledge before intervention, while majority $(62.5 \%)$ of nurses and only (25.0\%) of traditional midwives scored very good knowledge after intervention. These are national policies that are recommended for safety birth, hence Sosa, Mary Ellen Burke study stated that Hand washing remains the cornerstone of prevention as transmission can occur directly from an asymptomatic colonized healthcare provider, other patients, or a communityacquired source [15].
Geberemariyam BS, Donka GM, Wordofa B [16] study reviled that Inadequate infection prevention knowledge and unsafe practices were frequent among study participants, reflecting a potentially common problem at public healthcare facilities in southeast Ethiopia. Healthcare workers have better knowledge and safer practices if they had received infection prevention training and had infection prevention guidelines in their workplace.

Concerning advice and guidance provided to the mother regarding hygiene, particularly perineal and vulvar care, significantly high proportions of nurses and midwives had poor scores of knowledge before the intervention. However, their knowledge significantly improved after intervention, many studies were mentioned poor knowledge and practice regarding perineal and vulvar care. As mentioned by Setyowati, Setyowati; Rosnani, Rosnan [17] whose stated that Mothers' efforts to solve postpartum problems cannot be separated from habit and the family's culture. Health workers need to facilitate maternal care with a family culture approach.

Perineal and vulvar care was done by using herbal medicine and home remittance, so more research is needed.

Safety Management reduces maternally and mortality, regarding postnatal sepsis Nursing assessment and management practice respondents, reflect About $(20.8 \%)$ demonstrated poor practice and only a few $(12.5 \%)$ of respondents demonstrated very good practice, while nearly $(82.6 \%)$ demonstrated very good practice after demonstration of the program. Same a study done by Geberemariyam BS, Donka GM, Wordofa B [18] stated that Inadequate infection prevention knowledge and unsafe practices were frequent among study participants, reflecting a potentially common problem at public healthcare facilities in southeast Ethiopia.

Regarding essential nursing skills need, like Skills full setting up and monitoring an intravenous infusion only a few $(12.5 \%)$ demonstrated poor practice and $(50.0 \%)$ of respondents demonstrated very good practice, while nearly $(91.3 \%)$ demonstrated very good practice after demonstration of the program, and regarding Skills full taking blood samples for analysis, only a few (13\%) demonstrated poor practice and $(47.8 \%)$ of respondents demonstrated very good practice, while nearly $(87.0 \%)$ demonstrated very good practice after demonstration of the program. Same things regarding skills full taking a midstream specimen of urine, About $(39.1 \%)$ demonstrated good practice and $(26.1 \%)$ of respondent demonstrated very good practice while $(34.8 \%)$ demonstrated bad practice, while nearly $(73.9 \%)$ demonstrated very good practice after demonstration of the program. On the other hand, regarding taking a high vaginal swab, majority (69.6\%) 
of respondents demonstrated bad practice and only (13.0\%) demonstrated good practice and (17.4\%) of respondent demonstrated very good practice while $(34.8 \%)$ demonstrated bad practice, while nearly $(60.9 \%)$ demonstrated very good practice after demonstration of the program. which indicate needs for such guidelines and more training practice. This mentioned by Tan and Hannah [19] stated that Infection during labor and in the postpartum period can be reduced through aseptic delivery practices and careful attention to risk factors for infection, including excessive vaginal examinations, premature rupture of membranes, and prolonged labor. Induction of labor in cases of uncomplicated prelabour rupture of membranes has been shown to reduce maternal and neonatal infection.

The prevention control measurement play a cornerstone role in postnatal management regarding following universal precautions for prevention of infection in postnatal ward, $(45.5 \%)$ demonstrated bad practice and only a few $(4.5 \% \%)$ of respondent demonstrated very good practice which changed significantly to (43.5\%) Good Practice and $(56.5 \%)$ Very Good Practice. This reported by Hussein $\mathrm{J}$ et al., [20] that specific interventions necessary for the prevention and treatment of infection are good hand hygiene, use of the antiseptic solution and appropriate antibiotic coverage. Increasing concerns of the hospital and health care-associated with infection control are currently recorded in many medical disciplines even in high income industrialized countries.

The reason behind that mostly because of lack of in-service training inadequate supply of consumables, poor supervision and absence of a hospital's policy on infection control this similar to a study of Brisibe S, Ordinioha B, Gbeneolo PK [21] in Nigeria, whose find that The reasons given by the respondents in UPTH for non-adherence to the infection control policy include poor supervision (39.39\%) and lack of in-service training $(21.21 \%)$, while the respondents in BMSH gave reasons that include inadequate supply of consumables (34.29\%) and absence of a hospital's policy on infection control $(22.88 \%)$.

The nutritional status of the woman has a direct impact on maternal and health, healing of the wound, increasing immunity and prevention of infection in the puerperium. Selected interventions and dietary advice can affect a woman's nutritional status. The present study mentioned that Concerning knowledge regarding advice should be provided to the mother regarding nutrition during the postpartum period $(34.6 \%)$ of nurses midwives had poor score of knowledge before the intervention, while the great majority $(80.0 \%)$ of them and only have of traditional midwives scored very good knowledge after intervention. this limited knowledge among nurses and midwives may suggest the need to review the current pre-service curricula to assess how adequately it prepares midwife with skills in various obstetric care. a similar study in Asmara by Ghebreyohans, Ghidey [22] reviled that The percentages of knowledge in recognizing the necessary nutrients ranged from $87.6 \%$ for carbohydrates to $46 \%$ for minerals.

Regarding advice provided to the mother having postnatal sepsis regarding child care, the present study assigned that there is a highly significant score of knowledge in post-intervention compared with before intervention with a statistically significant difference. The lack of guidance and limited information that currently exists among nurses who provide maternal health services may indicate that baby care is given low priority. It is therefore important to ensure that midwives are equipped with the knowledge to provide advice, guidance, and counseling to the woman. That is prescribed in the study of Ghebreyohans, Ghidey [22] whose stated that The percentages of correct knowledge regarding first baby bath, frequency of breastfeeding, umbilical care, duration of exclusive breastfeeding, need and purpose of the vaccine was 40.1, 81.9, 77.4, 94.8 , and $99.2 \%$ respectively. The mean PNC knowledge score was $24.89 / 60$. The score of knowledge on postnatal care was found to significantly differ across the categories of residence $(p<0.001)$ [22] this means that the midwife was not exposed to this kind of information, which may call for a review of the midwifery curricula.

\section{CONCLUSION}

Based on the findings of the present study it can be concluded that:

1. A majority of nurses and midwives have some knowledge in postpartum infection management but do not always practice what they know because of shortage of facilities and inadequate supply.

2. The majority of nurses and midwives were lacked of the essential knowledge regarding infection control measurements

3. Culture and believes contribute nurses and midwives' behaviors

4. The nurses and midwives had high knowledge score in the post-test compared with pre-test and they demonstrated good practice which indicated that the postpartum infection management training program was effective.

\section{RECOMMENDATIONS}

Based on the study findings the following recommendations are suggested:

1. Every delivery, including those that take place in the home, should be assisted by a skilled birth attendant (a midwife, physician, or nurse) who has been trained to proficiency in basic techniques for a clean and safe delivery. 
2. On going training and refresher courses to upgrade nurses' and midwives' knowledge and skills on Postpartum care which should emphasize the prevention, timely recognition, and treatment of infection.

3. Reviewing of current pre-service curricula to assess how adequately it prepares nurses and midwives with skills in various obstetric cares, still there is a need for a better access to quality and safe health technologies.

4. Empower nurses and midwife to play and implement their role as health educator, counselor to help post-natal mothers to improve their knowledge regarding postpartum infections.

5. Increased and sustained resources by governments and partners to improve infection control measurements and provide adequate facilities and supplies.

6. Improved generation of evidence through information systems and research, further extensive researches are needed.

\section{REFERENCES}

1. Hill, K., AbouZahr, C., \& Wardlaw, T. (2001). Estimates of maternal mortality for 1995. Bulletin of the World Health Organization, 79, 182-193.

2. Weil, O., \& Fernandez, H. (1999). Is safe motherhood an orphan initiative? The Lancet, 354(9182), 940-943.

3. Loudon, I. (2000). The tragedy of childbed fever.

4. Lucas, A. O., Stoll, B. J., \& Bale, J. R. (Eds.). (2003). Improving birth outcomes: meeting the challenge in the developing world. National Academies Press.

5. Ounsa, M. A. A. E., \& Mohamed, E. Y. (2011). Maternal Mortality in Ribat University Hospital, Khartoum, Sudan: Seven years of experience. Sudan Journal of Medical Sciences, 6(4).

6. Mohammed, A. A., Elnour, M. H., Mohammed, E. E., Ahmed, S. A., \& Abdelfattah, A. I. (2011). Maternal mortality in Kassala State-Eastern Sudan: community-based study using reproductive age mortality survey (RAMOS). BMC pregnancy and childbirth, 11(1), 102.

7. Li, X. F., Fortney, J. A., Kotelchuck, M., \& Glover, L. H. (1996). The postpartum period: the key to maternal mortality. International Journal of Gynecology \& Obstetrics, 54(1), 1-10.

8. Labib, A. (2020). Sepsis Care Pathway 2019. Qatar medical journal,2019 (2-Qatar Critical Care Conference Proceedings), 4.

9. Cunha, M. R., Padoveze, M. C., \& Nichiata, L. Y. I. (2018). Identification of post-cesarean surgical site infection: nursing consultation. Revista brasileira de enfermagem, 71, 1395-1403.
10. Burlinson, C. E. G., Sirounis, D., Walley, K. R., \& Chau, A. (2018). Sepsis in pregnancy and the puerperium. International journal of obstetric anesthesia, 36, 96-107.

11. Simon, E. G., \& Laffon, M. (2015). Maternal care after vaginal delivery and management of complications in immediate post-partum-Guidelines for clinical practice. Journal de gynecologie, obstetrique et biologie de la reproduction, 44(10), 1101-1110.

12. Maclean, G. D. (2003). The challenge of preparing and enabling 'skilled attendants' to promote safer childbirth. Midwifery, 19(3), 163-169.

13. Mirmolaei, S. T., Valizadeh, M. A., Mahmoodi, M., \& Tavakol, Z. (2014). Comparison of effects of home visits and routine postpartum care on the healthy behaviors of Iranian low-risk mothers. International journal of preventive medicine, 5(1), 61-68.

14. Willson, J. R. (1988). The conquest of cesarean section-related infections: a progress report. Obstetrics and gynecology, $72(3 \mathrm{Pt} 2), 519$ 532.

15. Sosa, M. E. B. (2016). Group A streptococcal infection in pregnancy and the puerperium. The Journal of perinatal \& neonatal nursing, 30(2), 124-130.

16. Rafferty, E., McDonald, W., Qian, W., Osgood, N. D., \& Doroshenko, A. (2018). Evaluation of the effect of chickenpox vaccination on shingles epidemiology using agent-based modeling. PeerJ, 6, e5012.

17. Setyowati, S., \& Rosnani, R. (2019). Women's efforts to solve postpartum problems based on the culture of South Sumatera. Enfermeria clinica, 29, 653-658.

18. Geberemariyam, B. S., Donka, G. M., \& Wordofa, B. (2018). Assessment of knowledge and practices of healthcare workers towards infection prevention and associated factors in healthcare facilities of West Arsi District, Southeast Ethiopia: a facilitybased cross-sectional study. Archives of Public Health, 76(1), 69.

19. Tan, B. P., \& Hannah, M. E. (2000). Oxytocin for prelabour rupture of membranes at or near term (Cochrane Review). The Cochrane Library, 3.

20. Hussein, J., \& Walker, L. (2010). Puerperal sepsis in low and middle income settings: past, present and future. Maternal and infant deaths: chasing millennium development goals, 4, 131-147.

21. Hinkson, L. (2016). The incidence of surgical site infection in Caesarean Sections with the use of a plastic sheath wound retractor compared to the traditional self-retaining metal retractor (Doctoral dissertation).

22. Ghebreyohans, G. (2011). Midwifery Care Experiences of Mothers during Labor and Delivery at Orota Maternity National Referral Hospital in Asmara, Eritrea. 